\title{
Litiasis biliar pediátrica en una población de alta prevalencia
}

\author{
PAUL HARRIS D. ${ }^{1}$, BERNARDITA CHATEAU I. ${ }^{1}$, JUAN FRANCISCO MIQUEL P. ${ }^{2}$ \\ 1. Departamento de Pediatría, Facultad de Medicina, Pontificia Universidad Católica de Chile. \\ 2. Departamento de Gastroenterología, Facultad de Medicina, Pontificia Universidad Católica de Chile.
}

\begin{abstract}
Childhood Cholelithiasis in a high prevalent population

Bile duct disease and gallstone (cholelithiasis) have been considered an uncommon cause of acute abdominal pain in children compared to adults. However, there are significant differences with the adult gallstone disease: increased proportion of patients with an underlying condition, higher incidence of acalculous cholecystitis and lower frequency of choledocolithiasis. With ultrasound examination, it is possible to detect early gallstone in the fetal period and in asymptomatic patients, explaining the increase in gallstone incidence. This disease is more common than suggested in western literature and its diagnosis is increasing as well. The high prevalence of gallstone in adult population in Chile creates an ideal setting for cholesterol and gallstone candidate gene testing. Although the clinical diagnosis of gallstone is simple, there is no consensus about the best therapy in children, explained partially by the lack of knowledge of the natural history of the disease. The role of gallstone disease acquired early in life in gallbladder carcinoma deserves special attention.

(Key words: Cholelithiasis, children, gallstone).

Rev Chil Pediatr 2007; 78 (5): 511-518
\end{abstract}

\section{RESUMEN}

Las enfermedades de la vía biliar y los cálculos vesiculares (colelitiasis) han sido consideradas como una causa poco frecuentes de dolor abdominal agudo en niños, a diferencia de lo que ocurre en adultos. Sin embargo, existen diferencias sustanciales con el adulto como son una mayor proporción de casos con causa identificable, mayor incidencia de colecistitis sin cálculos y menor frecuencia de coledocolitiasis. Con la masificación del ultrasonido es posible detectar litiasis biliar en etapa intrauterina, y en pacientes asintomáticos, lo que estaría explicando, en parte, el aumento de su incidencia. La litiasis biliar es más frecuente de lo sugerido clásicamente en la literatura occidental y su diagnóstico está en aumento. La alta prevalencia de colelitiasis en población adulta chilena, posicionan a nuestro país como un escenario ideal para el estudio de genes candidatos asociados a la etiopatogénesis de la litiasis de colesterol. Si bien el diagnóstico de la litiasis es relativamente simple, no hay consenso en la terapia de elección, lo que se explica fundamentalmente por una historia natural desconocida. El rol de la litiasis de colesterol a edades tempranas en la etiopatogénesis de procesos neoplásicos de la vía biliar merece especial atención.

(Palabras clave: Colelitiasis, niños, cálculos).

Rev Chil Pediatr 2007; 78 (5): 511-518

Trabajo recibido el 4 de abril de 2007, devuelto para corregir el 11 de junio de 2007, segunda versión el 17 de julio de 2007 , aceptado para publicación el 8 de septiembre de 2007.

Correspondencia a:

Dr. Paul Harris D.

E-mail:pharris@med.puc.cl 


\section{Introducción}

Litiasis biliar se define como la presencia de material sólido, cálculo o barro, en el tracto biliar, generalmente en la vesícula biliar (VB). El concepto de enfermedad vesicular, por otra parte, indica cambios funcionales y/o morfológicos (inflamación o fibrosis) en la $\mathrm{VB}$, secundarios al desarrollo de bilis con capacidad para formar cálculos (bilis litogénica) y/o asociados a la presencia de litiasis vesicular propiamente tal. Las enfermedades de la vía biliar y los cálculos vesiculares (colelitiasis) han sido consideradas como una causa poco frecuente de dolor abdominal agudo en niños, a diferencia del adulto en quienes esta patología es una de las enfermedades digestivas más prevalentes y es causa importante de morbilidad en occidente, especialmente en nuestro país. Existen, además, diferencias sustanciales con el adulto como son una mayor proporción de casos con causa identificable, mayor incidencia de colecistitis sin cálculos y menor frecuencia de coledocolitiasis. Actualmente, con la masificación del ultrasonido es posible detectar litiasis biliar tan tempranamente como en etapa intrauterina, y en pacientes asintomáticos como hallazgo ecográfico, lo que estaría explicando, en parte, el aumento de la incidencia clínica de esta patología en pediatría. Si bien la historia natural de esta enfermedad y las conductas terapéuticas están relativamente bien establecidas en adultos, desconocemos cual es la historia natural de la colelitiasis en niños y por lo tanto, las conductas médicas a seguir.

\section{Objetivo y Metodología}

El objetivo de esta revisión es definir conceptos sobre litiasis biliar en niños, analizar prevalencias y características de esta enfermedad tanto en nuestro país como en el extranjero, y a partir del conocimiento actual sugerir conductas a tomar basadas en la evidencia recolectada a través de los años. Para la presente revisión del tema se estudiaron artículos sobre litiasis biliar en niños en una búsqueda de artículos publicados en PubMed (http://www.ncbi. nlm.nih.gov/entrez/query.fcgi? $\mathrm{DB}=$ pubmed) en los últimos 25 años y en Scielo (http://www. scielo.cl/scielo.php?lng=es) en los últimos 6 años, tanto en revistas nacionales y extranjeras de corriente principal. Se utilizaron las siguientes palabras claves: infant/pediatric cholelitiasis, infant/pediatric biliary stones (para PubMed) y colelitiasis infantil/pediátrica, cálculo biliar pediátrico/infantil (para Scielo). No hubo restricción por idioma. La búsqueda se realizó en Marzo 2006. La búsqueda de referencias relacionadas a la historia de los primeros reportes se hizo en forma manual y en base a un archivo digital recientemente disponible de la Sociedad Chilena de Pediatría desde 1930.

\section{Magnitud del problema}

Población adulta. Sólo en Estados Unidos hay aproximadamente 25 millones de adultos con colelitiasis, presentando un total de $800 \mathrm{mil}$ casos nuevos por año. La colecistectomía es la $3^{\mathrm{a}}$ cirugía en frecuencia, con un número total de $500 \mathrm{mil}$ al año. Adicionalmente, se reportan unas 6000 muertes anuales y se considera que esta patología da cuenta de $2,5 \%$ de todos los costos anuales en salud ${ }^{1}$. En Chile, la prevalencia global en población mayor a 20 años se estima 17 y $30 \%$, para hombres y mujeres, respectivamente, lo que constituye un universo de aproximadamente 2300000 personas adultas con antecedentes de colelitiasis o con patología biliar latente asintomática. Se describe que un 30 a $50 \%$ de los pacientes son sintomáticos, de estos, el 50\% son sometidos a colecistectomía, y de las colecistectomías, la mitad son de urgencia y un $40 \%$ laparoscópicas ${ }^{2}$.

Las cifras de prevalencia están basadas en estudios poblacionales realizados con ultrasonido en los que se describe que las mujeres tienen una mayor frecuencia de colelitiasis que los hombres y que la frecuencia de esta patología aumenta con la edad, en ambos sexos ${ }^{3}$. En Chile y en etnias nativas de América, como los Indios Pima en Estados Unidos, la prevalencia de litiasis biliar es mayor que en la población general. En ellos, la bilis litogénica puede ser detectada desde los 10 años, con una prevalencia de litiasis en aumento, desde un $10 \%$ en la adolescencia hasta un $70 \%$ a los 40 años en 
mujeres (en hombres 2 y $25 \%$ respectivamente $)^{4}$. En Chile los primeros datos están basados en estudios de material de autopsia y demuestran frecuencias que van desde un $25 \%$ a la edad de 20 años hasta un $78 \%$ sobre los 70 años para mujeres y bastante menor para hombres $^{5}$. El estudio realizado por Miquel y cols, entre los años 1993 y 1995, describió una prevalencia global sobre 20 años, de 17 y $30 \%$ en hombres y mujeres, respectivamente. También demostró que la frecuencia en la población chilena mapuche fue significativamente mayor que en la población chilena hispana, y también mayor que en población Maori, poniendo de relevancia el factor genético ${ }^{6}$. En una perspectiva americana, los Indios Pima en Estados Unidos y los Mapuches en Chile presentan la mayor prevalencia regional, superando por mucho a la población hispana chilena, mexicanaamericana, mexicana, peruana, cubana, portorriqueña, brasilera, angloamericana y española ${ }^{6}$.

Población infantil. La colelitiasis en niños ha sido considerada una entidad poco frecuente y existen pocos estudios al respecto. En 1874 se reporta el primer caso de colelitiasis infantil por Gibson, luego, en 1928 Potter revisa la primera serie con 228 casos publicados ${ }^{7}$. Una revisión de la literatura en Chile muestra que en el año 1930 aparecen las dos primeras discusiones de casos clínicos de litiasis biliar pediátrica por Armando y Gandulfo, en publicaciones separadas $^{8,9}$. Desde 1931 hasta 1970 logramos rescatar 11 publicaciones de casos pediátricos aislados de litiasis biliar, y a partir del año 1970 tres series mayores ${ }^{10-12}$.

Con el uso masivo del ultrasonido, la litiasis biliar es diagnosticada más frecuente y más tempranamente, siendo posible detectarla in útero y neonatos. La incidencia en niños pareciera estar en aumento, aún cuando la información es segmentaria. Existe una clara distribución por sexo, lo que lo transforma en un factor de riesgo para la colelitiasis, sólo a partir de la edad puberal, como fue demostrado en la década de los 60 por Nilssonn y cols, reportando una diferencia entre niños y niñas que era sólo evidente a partir de los 11 y 12 años, en la cual se produce un considerable aumento de casos femeninos ${ }^{13}$.

\section{Tipo y composición de los cálculos}

Un aspecto diferente en niños en comparación con adultos es la composición de los cálculos. De acuerdo al contenido de colesterol, los cálculos pueden ser clasificados como cálculos de colesterol, cuando el porcentaje de colesterol es mayor a un $50 \%$, cálculos de pigmento, cuando el porcentaje de colesterol es menor a un $30 \%$, y mixtos cuando el porcentaje de colesterol es entre un 30 y $50 \%$. Adicionalmente los cálculos de pigmento pueden ser clasificados como negros o cafés (figura 1).

Cálculos de pigmento negro. Estos cálculos son negros como alquitrán, duros, aspecto brillante y cristalino. Se presentan en formas múltiples, son irregulares y menores a $5 \mathrm{~mm}$. Su composición está dada en un $40 \%$ por polímeros de pigmento, 15 a $60 \%$ por sales de calcio (fosfatos, carbonatos), $2 \%$ de colesterol y $30 \%$ de otros componentes. El 50\% de ellos son radiopacos. La localización habitual corresponde a la VB y a los conductos intrahepáticos. Se considera que corresponden al 25\% de los cálculos encontrados en actos quirúrgicos en los Estados Unidos, presentando una diferencia por género y raza.

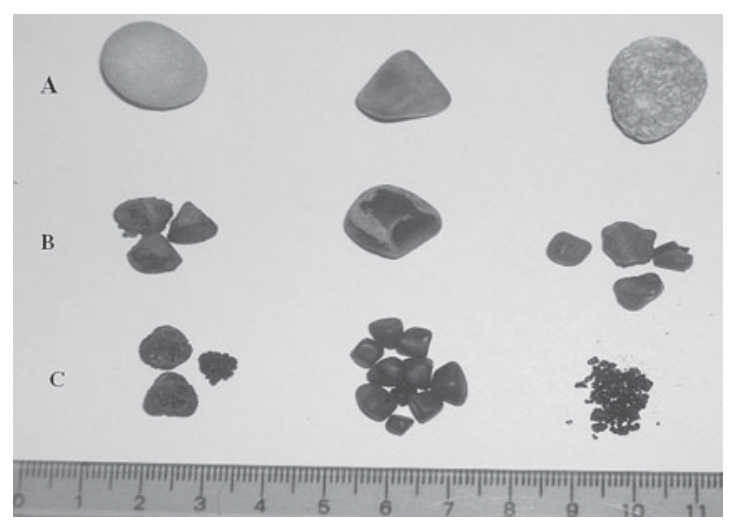

Figura 1. Fotografía que muestra distintos aspectos macroscópicos (forma, tamaño comparativo, color, superficie) de cálculos de colesterol (fila A), cálculos mixtos (fila B) y cálculos pigmentarios (fila $\mathrm{C}$ ) de pacientes chilenos sometidos a colecistectomía. 
Los grandes factores de riesgo en la patogenia de los cálculos negros son la hemólisis, la cirrosis hepática y la nutrición parenteral. No tienen relación con infección de la VB o de la vía biliar.

La hemólisis con el exceso de bilirrubina no conjugada determina una conjugación incompleta de la bilirrubina, generando actuando ambas como un co-precipitante en forma de bilirrubinato de calcio, favoreciendo la polimerización y unión a mucina producida normalmente por la vesícula. En la cirrosis, entre otros factores, disminuye la secreción de sales biliares lo que afecta la solubilidad del pigmento y su unión a calcio. En el caso de la nutrición parenteral total, participan factores similares a los mencionados, donde se forma un barro biliar, que es un sedimento de cristales de colesterol más gránulos de bilirrubina en una matriz de gel-mucosa. En esta situación, el cálculo contiene un mayor contenido de colesterol que los cálculos negros clásicos, pero no tienen ácidos grasos que caracterizan al cálculo café. La aparición de barro biliar se ha observado al cabo de cuatro a seis semanas de uso, y siempre que el uso de nutrición parenteral total se asocie a ayuno.

Cálculos de pigmentos café. Estos cálculos de color café anaranjado, se presentan en formas múltiples, de 10 a $30 \mathrm{~mm}$, de consistencia regular y suave. Su composición está determinada en un $60 \%$ por bilirrubinato de calcio, un $15 \%$ por jabones de ácido grasos con calcio (palmetato, estearatos), 15\% por colesterol y $10 \%$ otros componentes. Estos cálculos son radiolúcidos, localizados preferentemente en el colédoco y frecuentes en Oriente, mucho más raro en Occidente. Han sido asociados con fenómenos obstructivos crónicos o cuerpo extraño, como infección crónica de la vía biliar por nematodos, colangitis crónica, material de sutura retenido, estenosis de la vía biliar y colangitis esclerosante.

La localización de estos cálculos es en la vía biliar intra y extrahepática, incluso en ausencia de VB y representan la mayoría de los cálculos recurrentes después de la colecistectomía. La composición bioquímica varía dependiendo de la localización, mientras más arriba en la vía biliar, mayor proporción de colesterol y menos de bilirrubina. En su patogénesis participan enzimas ( $\beta$-glucoronidasa), de los tejidos o bacterias, que deconjugan la bilirrubina conjugada, y fosfolipasa A1, que generan ácidos grasos a partir de lecitinas. La conjunción de esta doble acción enzimática lleva a la precipitación ductal de bilirrubina y de colesterol y la consiguiente formación de cálculos suaves, grasos y moldeados a la vía biliar.

Cálculos de colesterol. Estos cálculos de aspecto amarillo blanquecinos, son de consistencia dura, cristalinos, a veces laminados y con un núcleo oscuro. Pueden ser múltiples (pequeños y facetados) o solitarios (de mayor tamaño, 2 a $4 \mathrm{~cm}$ y redondeados). Por definición, su composición esta dada por más de un $50 \%$ de colesterol monohidrato y el restante $50 \%$ por glicoproteínas y sales de calcio. Son radiolúcidos y generalmente se ubican en la VB y en el colédoco.

$\mathrm{Su}$ patogénesis se puede esquematizar en tres estadios. En el estadio químico, la bilis se sobresatura con colesterol y no puede ser solubilizada con sales biliares y lecitina (tabla 1). En el estadio físico, el colesterol precipita como microcristal ya que es insoluble en agua y debe ser solubilizado por las sales biliares y por lecitinas. La capacidad de solubilización de la bilis es limitada y el exceso de colesterol puede ser mantenido solo temporalmente como micelas supersaturadas. Este equilibrio frágil está representado por el clásico diagrama en que una disminución en la concentración de sales biliares, o una disminución en la concentración de lecitinas, o un aumento en la concentración de colesterol lleva rápidamente a desplazar al colesterol de una fase líquida micelar a un estado de cristales de colesterol que favorece la precipitación para luego entrar en un estadio de crecimiento (1 a $2 \mathrm{~mm}$ por año) agravado por ectasia vesicular.

Composición de cálculos en la población chilena. En población adulta, Miquel ha reportado un $83 \%$ de cálculos de colesterol, un $12 \%$ de cálculos mixtos y $5 \%$ de cálculos de pigmento ${ }^{14}$. En pediatría escasos datos publicados en cuanto a composición bioquímica. En un repor- 
Tabla 1. Fisiopatología de la formación de bilis saturada con colesterol

\begin{tabular}{cll}
\hline Defecto en la secreción & Mecanismo & Ejemplos \\
\hline $\begin{array}{c}\text { Disminución en la secreción de SB* } \\
\text { Pérdidas excesivas }\end{array}$ & $\begin{array}{l}\text { Reducción en pool SB } \\
\text { Malabsorción que disminuye pool SB }\end{array}$ & $\begin{array}{l}\text { Resección ileal, enfermedad ileal, } \\
\text { fibrosis quística, defecto congénito } \\
\text { de transporte de SB }\end{array}$ \\
Síntesis defectuosa & $\begin{array}{l}\text { Síntesis disminuida en relación al } \\
\text { pool depletado }\end{array}$ & $\begin{array}{l}\text { Defectos congénitos de síntesis de SB, } \\
\text { cirrosis }\end{array}$ \\
Aumento en la secreción de colesterol & $\begin{array}{l}\text { Exceso de síntesis de colesterol, de } \\
\text { la movilización tisular o aumento } \\
\text { en la ingesta }\end{array}$ & $\begin{array}{l}\text { Obesidad, drogas (hormonas), } \\
\text { hipolipoproteinemias }\end{array}$ \\
Ambos mecanismos & & Mayoría de los pacientes con cálculos \\
\end{tabular}

*SB, sales biliares.

te reciente de Harris y cols, se describe $47 \%$ de cálculos de colesterol y $53 \%$ de pigmento, en 26 niños evaluados post cirugía ${ }^{15}$.

\section{Factores genéticos}

Diferencias por género han sido descritas en litiasis biliar de adultos ${ }^{6}$. Sin embargo, en niños prepuberales, independiente de la naturaleza del cálculo (colesterol o pigmento) no hay diferencias en género ${ }^{16-19}$. Los niños con litiasis de colesterol presentan frecuentemente sobrepeso u obesidad, lo que sugiere que factores distintos al sexo juegan un papel importante. La evaluación del componente genético en niños es difícil considerando aun el número bajo de niños con litiasis. En adultos, sobre el $80 \%$ de los pacientes litiásicos, tiene litiasis de colesterol. La enfermedad en ellos es más común en mujeres y se ha asociado a factores de riesgo que alteran el metabolismo del colesterol como número de embarazos, obesidad y diabetes mellitus $^{6,20}$. Es interesante notar que sobre el 50\% de los niños con litiasis biliar tiene familiares con litiasis ${ }^{6}$. Estos niños que desarrollan litiasis de colesterol temprano en la vida probablemente representan un subgrupo de familias chilenas con una alta expresión de genes involucrados en esta común y compleja enfermedad ${ }^{6,14}$. Son estos niños pequeños, que como caso índice, y sus familiares los que permitirán la búsqueda y análisis de genes candidatos involucrados en el metabolismo del colesterol y patogénesis de la litiasis.

\section{Elementos clínicos y de diagnóstico}

La litiasis biliar en niños ha sido descrita como una entidad poco frecuente y clásicamente asociada a enfermedades hemolíticas, nutrición parenteral, malformaciones congénitas de la vía biliar y obesidad. La mayoría de los textos clásicos describen que los cálculos son pigmentarios y radiopacos, y que la clínica es fundamentalmente un cuadro de cólico biliar.

En la tabla 2 se resume en forma esquemática los mayores factores de riesgo para cálculos de pigmento y de colesterol, como las aso-

Tabla 2. Asociaciones clínicas en la formación de cálculos

\begin{tabular}{ll}
$\begin{array}{l}\text { Cálculos de } \\
\text { Pigmento Negro }\end{array}$ & $\begin{array}{l}\text { Cálculos de } \\
\text { Colesterol }\end{array}$ \\
\hline Anemias hemolíticas & Fibrosis quística \\
1. Anemia células falciformes & $\begin{array}{c}\text { Enfermedad ileon } \\
\text { 2. Talasemias }\end{array}$ \\
3. Microesferocitosis & $\begin{array}{c}\text { 2. Intestino corto } \\
\text { NPT }\end{array}$ \\
Cirrosis & Defectos congénitos SB* \\
Infección de la vía biliar & \\
Inflamación de la vía biliar & \\
\hline
\end{tabular}

*SB, sales biliares. 
ciaciones clínicas más características. Sin embargo, los dos elementos más importantes al momento de analizar las asociaciones clínicas son el género y la edad. Clásicamente se planteó el género como un elemento importante en la litiasis en pediatría y de hecho así lo muestra el trabajo de Nilsson ${ }^{13}$. Posterior a esta observación, numerosos trabajos han demostrado que no hay una mayor prevalencia de litiasis en niños antes de la edad puberal. Unos de los estudios más significativos publicados fue el realizado por Reif en 1991, quien estudió 50 niños con diagnóstico ecográfico de litiasis biliar en un período de 10 años, con una edad promedio de 12,2 años, y demostró que no existía predominio de sexo cuando los pacientes eran analizados de acuerdo a los factores clínicos de riesgo. Así, en aquellos grupos con enfermedad hemolítica, nutrición parenteral, e incluso sin factores de riesgo no hubo predominio por sexo exceptuando el grupo de embarazadas adolescentes ${ }^{16}$.

Del punto del vista de las condiciones clínicas asociadas por edad, es necesario referirse al trabajo de Friesen ${ }^{17}$, la serie clínica más grande reportado a la fecha con un total de 693 casos de colelitiasis en niños, analizando las condiciones clínicas asociadas considerando el factor edad. En los menores de 12 meses de edad en más de un tercio de los casos no se identifica ninguna condición asociada mientras que la nutrición parenteral y cirugía abdominal previa se asocian en un $29 \%$ en ambos casos. En niños entre 1 y 5 años, la enfermedad hepatobiliar es la patología asociada más frecuente $(29 \%)$, le siguen en frecuencia cirugías abdominal y cardíaca con 21 y 14\%, respectivamente. En mayores de 6 años y adolescentes, las condiciones asociadas son embarazo y enfermedad hemolítica, con un 37 y $23 \%$, respectivamente.

Barro biliar como condición previa a la formación de litiasis. Un trabajo retrospectivo reciente de Wesdorp y cols, de 82 pacientes con colelitiasis, 75 presentaban barro biliar, 39\% tenía colelitiasis asociada a hemolisis y un 23\% no tenía ninguna asociación. En niños con barro biliar, la nutrición parenteral y la presencia de infección sistémica o uso de antibióticos fueron los posibles factores predisponentes ${ }^{21}$. En otro estudio, 75 niños con anemia de células falciforme, se estudiaron ecográficamente por síntomas abdominales vagos, demostrando que 17 de ellos tenían sólo barro biliar, 9 tenían barro biliar y cálculos siendo sometidos a colecistectomía, y 8 no tenían cálculos inicialmente, sin embargo, desarrollaron colelitiasis durante los siguiente 30 meses, sugiriendo colecistectomía precoz en estos pacientes ${ }^{22}$. Un estudio de cohorte de 429 niños en Jamaica con anemia de células falciformes y ecografía anual desde los 5 años, pesquisó 17 niños sintomáticos que presentaron barro biliar, 12 de ellos desarrollaron litiasis biliar, 5 no presentaron litiasis, y el barro biliar se resolvió espontáneamente en 4 de ellos, de modo que en total sólo 4 de 81 presentaron síntomas y requirieron cirugía ${ }^{23}$. La diferencia entre ambos estudios puede estar dada básicamente por la población de referencia, variedad en la sintomatología, en el uso de ceftriaxona, factores dietéticos que en Estados Unidos hayan favorecidos el desarrollo de cálculos de colesterol, y eventualmente diferencias en la sensibilidad al dolor determinando la indicación de ecografía.

\section{Tratamiento}

Cálculos negros. El tratamiento de elección para este tipo de cálculos es la colecistectomía (calidad de evidencia II 3-III). Ni el tratamiento con fármacos orales ni la litotripsia ha sido efectivo. El futuro en el manejo de esta condición considera solventes de contacto como mono-octanoico con ácido etilenglicol tetracético.

Cálculos de colesterol. En un plano teórico, el tratamiento de elección en estos cálculos será la disolución con ácidos biliares orales como ácido quenodeoxicólico, ácido ursodeoxicólico o su combinación. Esta opción está limitada a pacientes asintomáticos o mínimamente sintomáticos con cálculos pequeños, lúcidos y flotantes, que corresponde aproximadamente al $30 \%$ de este tipo de condición, y requiere buena adhesión al tratamiento por al menos 1 a 2 años. En un estudio de Gamba y cols, en 15 
pacientes con edad promedio 7,8 años con cálculos radiolúcidos de menos de $10 \mathrm{~mm}$ y VB normal, se utilizó ácido ursodeoxicólico $10 \mathrm{mg} /$ $\mathrm{kg}$ /día por 1 año y se observó disolución ecográfica en 2 de ellos durante la terapia, reaparición de los cálculos al suspender el ácido ursodeoxicólico y en 5 niños los síntomas desaparecieron ${ }^{18}$. En el estudio de Harris y cols, de un total de 26 niños con litiasis biliar, en 7 se utilizó ácido ursodeoxicólico $30 \mathrm{mg} / \mathrm{kg} / \mathrm{día}$ dividido en 3 dosis y seguimiento por 6 meses, en 2 de estos niños no se logró disolución o disminución de tamaño del cálculo y se realizó colecistectomía $^{15}$.

La cirugía en niños con cálculos de colesterol está indicada en niños con recurrencia de crisis de dolor. Se ha discutido la indicación quirúrgica en pacientes asintomáticos dado el riesgo teórico de cáncer vesicular, considerando el riesgo familiar de cáncer vesicular ${ }^{24}$, la relación inversa entre colecistectomía y cáncer vesicular ${ }^{25} \mathrm{y}$ asociación entre colelitiasis y cáncer vesicular ${ }^{26,27}$.

Una situación especial es la indicación en menores de 1 año, en los que se debe tomar la precaución de un período de observación mayor a 1 año, considerando el mayor porcentaje de resolución espontánea en este grupo etario ${ }^{28}$.

La colecistectomía es una operación de baja mortalidad, pero no ausente de ella. En un estudio de Grosfeld y cols, de 169 colecistectomías, 143 fueron por laparotomía y 26 por laparoscopía, se constató una mortalidad de 1 paciente por sepsis $(0,6 \%)$, y morbilidad en 2 por neumonia, 3 por infección de herida operatoria, 2 por pancreatitis postoperatoria y 1 por íleo prolongado ${ }^{29}$. En una publicación nacional por Harris y cols, de 25 pacientes con litiasis biliar, se realizó colecistectomía en 15 (58\%), de ellos, $13(86,7 \%)$ fue laparoscópica y 2 $(13,3 \%)$ por laparotomía. Dos $(13,3 \%)$ pacientes con ácido ursodeoxicólico se operaron por no lograr disolución o disminución de tamaño ecográfico del cálculo en 6 meses. No se constataron complicaciones derivadas de la cirugía. La composición bioquímica de los cálculos mostró $8(53 \%)$ niños con cálculos de pigmento y 7 (47\%) con cálculos de colesterol. De los niños no operados, $6(54,5 \%)$ estuvieron en observación sin terapia médica, no presentaron compli- caciones y se mantuvieron en seguimiento por un promedio de 11,8 meses. Cinco (45,5\%) niños aún continuaban con terapia médica con ácido ursodeoxicólico $30 \mathrm{mg} / \mathrm{kg}$ /día y en seguimiento clínico ${ }^{15}$.

La escasa literatura no permite establecer un consenso en la terapia de elección, lo que se explica fundamentalmente por una historia natural desconocida.

\section{Conclusiones}

La litiasis biliar es más frecuente de lo sugerido clásicamente en la literatura occidental y su diagnóstico está en aumento en parte por la masificación del uso diagnóstico de ultrasonido abdominal en niños con síntomas digestivos de variada naturaleza incluyendo aquellos niños con Tratornos Digestivos Funcionales. La alta prevalencia de colelitiasis en población adulta chilena, posicionan a nuestro país como un escenario ideal para el estudio de genes candidatos asociados a la etiopatogénesis de la litiasis de colesterol. Si bien el diagnóstico de la litiasis es relativamente simple, no hay consenso en la terapia de elección, lo que se explica fundamentalmente por una historia natural desconocida. El rol de la litiasis de colesterol a edades tempranas en la etiopatogénesis de procesos neoplásicos de la vía biliar merece especial atención.

\section{Referencias}

1.- Shaffer EA: Gallbladder disease. Gallstone disease. En: Walker W, Durie P, Hamilton R, Watkins J eds. Pediatric gastrointestinal disease: Pathophysiology, diagnosis, management. Eds: BC Decker Inc 1991; 1152-70.

2.- Pérez-Ayuso RM, Hernández V, González B, et al: Historia natural de la colelitiasis. Incidencia de colecistectomía en un área urbana y una rural mapuche, en la última década. Rev Méd Chile 2002; 130: 72330 .

3.- Shaffer EA, Small DM.: Gallstone disease: pathogenesis and management. Curr Probl Surg 1976; 13: 3-72.

4.- Bennion LJ, Knowler WC, Mott DM, Spagnola AM, Bennett PH: Development of lithogenic bile during puberty in Pima indians. N Engl J Med 1979; 19: 873-6. 
5.- Marinovic I, Guerra C, Larach G: Incidence of cholelithiasis in autopsy material and analysis of the composition of the calculi. Rev Méd Chile 1972; 100: 1320-7.

6.- Miquel JF, Covarrubias C, Villaroel L, et al: Genetic epidemiology of cholesterol cholelithiasis among chilean hispanics, amerindians, and maoris. Gastroenterol 1998; 115: 937-46.

7.- Potter AH: Gallbladder disease in young subjects. Surg Gynecol Obstet 1928; 46: 795-808.

8.- Armando A: Colecistitis en una niña de 9 años. Rev Chil Pediatr 1930; 1: 636.

9.- Gandulfo J: Dos casos de litiasis biliar en niños. Rev Chil Pediatr 1930; 1: 547.

10.- Paredes G, Barraza P, Veloso A, Rojas E, Espinoza J: Colecistitis y colelitiasis en niños (nuestra experiencia en 150 casos operados). Rev Chil Pediatr 1970; 42: 1053-62.

11.- Cerda J, Torres F, Aguirre J, Aldunate G, David W, Vildosola $C$ : Colecistitis y colelitiasis en niños. Rev Chil Pediatr 1976; 47: 133-7.

12.- Guzmán S, Arroyo C, López F, et al: Colecistectomía laparoscópica en menores de 15 años de edad. Rev Chil Cir 1997; 49: 507-9.

13.- Nilsson S: Gallbladder disease and sex hormones. Acta Chir Scand 1966; 132: 275-9.

14.- Miquel JF, Núñez L, Amigo L, et al: Cholesterol saturation, not proteins or cholecystitis, is critical for crystal formation in human gallbladder bile. Gastroenterol 1998; 114: 1016-23.

15.- Harris $P$, Chateau B, Miquel JF, et al: Litiasis biliar en niños: un estudio clínico-morfológico. Rev Méd Chile 2003; 131: 37-45.

16.- Reif S, Sloven D, Lebenthal E: Gallstones in children: Characterization by age, etiology, and outcome. Am J Dis Child 1991; 145: 105-8.

17.- Friesen $C$, Roberts $C$ : Cholelithiasis. Clinical characteristics in children. Case analysis and literature review.
Clin Pediatr 1989; 28: 294-8.

18.- Wesdorp I, Bosman D, De Graaf A, Aronson D, Van Der Blij F, Taminiau J: Clinical presentation and predisposing factors of cholelithiasis and sludge in children. J Pediatr Gastroenterol Nutr 2000; 3: 411-7.

19.- Winter SS, Kinney TR, Ware RE: Gallbladder sludge in children with sickle disease. J Pediatr 1994; 125: 7479.

20.- Walker TM, Serjeant GR: Biliary sludge in sickle cell disease. J Pediatr 1996; 129: 443-5.

21.- Gamba PG, Zancan L, Midrio P, Muraca M, Vilei M, Talenti E, et al: Is there a place for medical treatment in children with gallstones?. J Pediatr Surg 1997; 32: 476-8.

22.- Misra S, Chaturvedi A, Misra NC, Sharma ID: Carcinoma of the gallbladder. Lancet Oncol 2003; 4: 16776.

23.- Serra I: Has gallbladder cancer mortality decrease in Chile? Rev Méd Chile 2001; 129: 1079-84.

24.- Lowenfels AB, Lindstrom CG, Conway MJ, Hastings PR: Gallstones and risk of gallbladder cancer. JNCI 1985; 75: $77-80$

25.- Su WC, Chan KK, Lin $P W$, et al: A clinical study of 130 patients with biliary tract cancers and periampullary tumors. Oncology 1996; 53: 488-93.

26.- St-Vil D, Yazbeck S, Luks FI, Hancock BJ, Filiatrault D, Youssef S: Cholelithiasis in newborns and infants. J Pediatr Surg 1992; 27: 1305-7.

27.- Grosfeld J, Rescorla F, Skinner M, West K, Scherer LR: The spectrum of biliary tract disorders in infants and children. Experience with 300 cases. Arch Surg 1994; 129: 513-20.

28.- Schrimer $W$, Grisoni E, Gauderer $W$ : The spectrum of cholelithiasis in the first year of life. J Ped Surg 1989; 24: 1064-7.

29.- Sama C, Labate AM, Taroni F, Barbara L: Epidemiology and natural history of gallstone disease. Semin Liver Dis 1990; 10: 149-58. 